\title{
DANOS DA MOSCA-BRANCA BEMISIA TABACI (GENN.) E DISTRIBUIÇÃO VERTICAL DAS NINFAS EM CULTIVARES DE SOJA EM CASA DE VEGETAÇÃO
}

\author{
R. Suekane, P.E. Degrande, I.S. de Lima Junior, M.V.B.M. de Queiroz, E.R. Rigoni
}

Universidade Federal da Grande Dourados, Faculdade de Ciências Agrárias, Rod. Dourados-Ithaum, km 12, CEP 79804-970, Dourados, MS, Brasil. E-mail: izidro.lima@ifms.edu.br

\section{RESUMO}

\begin{abstract}
A mosca-branca Bemisia tabaci causa danos em plantas de soja em decorrência da sucção de seiva, transmissão de vírus e favorecimento da fumagina, que afetam o desenvolvimento e a produção da cultura. O objetivo deste trabalho foi avaliar os danos diretos da mosca-branca e a distribuição vertical de ninfas em cultivares de soja em condições de casa de vegetação. O delineamento experimental foi de blocos ao acaso, com 10 cultivares no esquema fatorial $10 \times 2$ (com e sem infestação) e 4 repetições. Contabilizou-se o número de ovos e ninfas em todos os folíolos e, separadamente, em um folíolo do terços superior, médio e inferior das plantas Os índices de produtividade analisados foram: número de vagens por planta e de grãos por vagem, massa de 100 grãos e massa total de duas plantas. Os dados foram submetidos à análise de variância e ao teste $\mathrm{F}(\alpha<0,05)$ e as médias comparadas pelo teste de Tukey ao nível de $5 \%$ de probabilidade, obtendo-se as diferenças mínimas significativas entre os tratamentos. As cultivares estudadas não foram afetadas pelo ataque de $B$. tabaci nos níveis de infestação registrados. CD 219 RR foi o cultivar mais infestada por ninfas de mosca-branca. Para melhorar a eficiência e o uso de tempo nas avaliações, em estudos em casa de vegetação, indica-se que estas sejam realizadas nos terços superior e médio das plantas.
\end{abstract}

PALAVRAS-CHAVE: Aleyrodidae, Glycine max, biótipo B, amostragem, resistência.

\section{ABSTRACT}

DAMAGE OF WHITEFLY (BEMISIA TABACI) (GENNADIUS, 1889) (HEMIPTERA: ALEYRODIDAE) AND VERTICAL DISTRIBUTION OF NYMPHS IN SOYBEAN GLYCINE MAX (L.) MERRIL CULTIVARS IN THE GREENHOUSE. The whitefly (Bemisia tabaci) causes damage in the soybean crop by sucking the plant sap, transmission of viruses and promoting sooty mold that affect the growing and the soybean production. The goal of this work was to evaluate the direct damage of the whitefly, and the vertical nymph distribution, on soybean cultivars under greenhouse conditions. The experimental design was randomized blocks with 10 cultivars in a $10 \times 2$ factorial scheme (infestation and without infestation) and 4 replications. Counts were made of the number of eggs and nymphs in the upper, middle and lower third of the plants. The productive indices were: number of pods per plant and grains per pod, weight of 100 grains, and weight of 2 plants. The data were submitted to variance analysis and $F$ test $(\alpha<0.05)$, and the means were compared by Tukey test at $5 \%$ probability, obtaining the least significant difference between treatments. The cultivars were not affected by the B. tabaci attack at the infestations levels registered. CD 219 RR was the cultivar most infested by the whitefly nymphs. For better efficiency and use of time in the evaluations, in greenhouse studies it is suggested that they be carried out in the upper and middle thirds of the soybean plants.

KEY WORDS: Aleyrodidae, Glycine max, biotype B, sampling, resistance.

\section{INTRODUÇÃO}

Amosca-brancaBemisiatabacibiótipoB(Hemiptera: Aleyrodidae) é praga de diversas culturas, sendo capaz de se alimentar de mais de 500 espécies de vegetais, principalmente algumas das famílias cucurbitáceas, solanáceas, brássicas, fabáceas, euforbiáceas, malváceas e plantas ornamentais (HAJı et al., 2004). Nesta relação, incluem os danos econômicos na soja, cuja área cultivada no País supera 23 milhões de hectares (ConselHo..., 2012) e, de acordo com a Associação... (2010), estima-se que na pauta de exportações do País os embarques deverão render, no total, US\$ 19,6 bilhões em 2011, onde a praga é uma ameaça à viabilidade dos negócios. No Brasil, severos surtos populacionais de $B$. tabaci biótipo $B$ ocorreram a partir do início dos anos 90 em plantas ornamentais e em lavouras de tomate e abóbora. 
Atualmente, vêm ocorrendo na região Centro-Oeste, onde tem causado danos à cultura da soja nas últimas safras agrícolas (DEGRANDE; VIVAN 2010).

Os prejuízos de B. tabaci na soja, em geral, decorrem da sucção da seiva, da excreção de um honeydew (melado) rico em açúcares que serve de substrato para o desenvolvimento do fungo fumagina (Capnodium spp., Capnodiaceae) capaz de afetar a fotossíntese, e pela transmissão de viroses (LoPEZ et al., 2008). Segundo LourEnÇão; NAGAI (1994), o biótipo B se caracteriza, entre outros fatores, por possuir maior quantidade de plantas hospedeiras, resistência a diversos inseticidas e capacidade de induzir desordens fisiológicas a certos tipos de hospedeiros.

No País, para diversas culturas, ainda não há estudos consolidados sobre o nível de controle desta praga. Um dos métodos de amostragem da praga, nas culturas de algodão, se baseia no exame criterioso de folhas do quinto nó, através da análise de presença/ ausência com contagem de adultos e ninfas grandes; o nível de controle definido é uma ninfa grande e 3-5 adultos por disco $\left(3,88 \mathrm{~cm}^{2}\right)$ de folha, ou $40 \%$ dos discos foliares infestados com uma ou mais ninfas grandes; estas indicações são baseadas no trabalho de DieHL et al. (1997a) e DieHL et al. (1997b) em estudos de amostragem binomial de ninfas e adultos realizado no Arizona (Estados Unidos).

Em paralelo, o conhecimento da distribuição vertical da espécie na planta torna-se um aspecto fundamental para as amostragens em que se trabalha com diversos genótipos em pesquisa, e com essas informações é possível realizar uma avaliação mais eficiente, reduzindo o tempo de amostragem (AzEVEDO; Bleicher, 2003).

Quanto ao controle da praga, uma solução em longo prazo é o manejo integrado, que utiliza como tática prioritária a resistência de cultivares ao hemíptero (FLINT; PARKS, 1990; Chu et al.; 2001). Para tal manejo, é necessária a utilização de táticas, como a escolha de cultivares de soja que sejam desfavoráveis para a colonização da B. tabaci (VAlLE; LOURENÇão, 2002; Lima et al., 2002). Existem alguns genótipos de soja relatados por VALLE; LOURENÇÃo (2012) que apontaram um certo grau de resistência a $B$. tabaci, podendo ser uma opção para uso nos programas de Manejo Integrado das Pragas.

O objetivo deste trabalho foi avaliar os danos da mosca-branca e a distribuição vertical das suas ninfas em cultivares de soja.

\section{MATERIAL E MÉTODOS}

O trabalho foi realizado em casa de vegetação, localizada no Município de Dourados, Estado de Mato Grosso do Sul. (latitude de 22 $13^{\prime} 16^{\prime \prime}$ S; longitude de $54^{\circ} 17^{\prime} 01^{\prime \prime} \mathrm{W}$; altitude de $430 \mathrm{~m}$ ).
As plantas foram cultivadas em vasos contendo $7,5 \mathrm{~kg}$ de solo oriundo de horizonte B de um Latossolo Vermelho distroférrico, cuja amostra coletada foi seca ao ar e, posteriormente, peneirada através de peneira com abertura de $2 \mathrm{~mm}$. Os valores da caracterização química das amostras para a recomendação de calagem e adubação necessária para a cultura foram obtidos através de interpretação de análise laboratorial. Cada unidade experimental consistiu de 2 vasos, cada qual continha duas plantas, totalizando 4 plantas por parcela. $\mathrm{O}$ delineamento experimental foi de blocos ao acaso, com 10 cultivares no esquema fatorial $10 \times 2$ (com e sem infestação) e quatro repetições. Os cultivares utilizadas foram: 1) BRS 245 RR, 2) BRS 243 RR, 3) BRS 246 RR, 4) BRS 239, 5) Embrapa 48, 6) CD 214 $\mathrm{RR}, 7) \mathrm{CD} 202$, 8) M 7908 RR, 9) NK 7059 RR, 10) CD 219 RR.

As irrigações atenderam as necessidades hídricas das plantas, tomando-se o cuidado de irrigar na base da planta, sem molhar a área foliar a fim de evitar o desenvolvimento de doenças. As plantas daninhas ocorrentes foram retiradas manualmente. Durante o desenvolvimento das plantas de soja ocorreram infestações de lagartas que foram eliminadas através de controle manual.

A criação de B. tabaci foi realizada em uma gaiola de estrutura de madeira, fechada com tecido branco "voal" que não permitia a entrada de outros insetos. A população inicial de mosca-branca foi obtida da criação de $B$. tabaci biótipo $B$ do laboratório de entomologia da Bayer CropSciense em Paulínia, SP. Para a criação e manutenção da população da mosca-branca utilizaram-se os seguintes hospedeiros cultivados em vasos dentro da gaiola de madeira: couve-manteiga (Brassica oleracea L. var. acephala), pingo-de-ouro (Duranta repens 'Aurea') e repolho (Brassica oleracea L. var capitata).

Para a infestação artificial, adultos de moscabranca oriundos da criação foram liberados dentro da casa de vegetação. A liberação dos adultos de moscas-branca foi realizada com os vasos das plantas hospedeiras colocados lado a lado a cada $50 \mathrm{~cm}$ de distância, totalizando 53 vasos, saturando o local com os insetos. Todas as unidades experimentais foram infestadas no dia 2 de dezembro de 2009, no momento em que os diferentes cultivares encontravam-se no estádio fenológico variando de V6 a V9 (segundo a escala de FeHR; CAviness, 1977). Para cada cultivar infestado havia uma testemunha (sem infestação). Nestas parcelas testemunhas de cada cultivar, foi realizado o controle químico preventivo, através da aplicação semanal do inseticida Piriprofixem 0,5 L ha $^{-1}$ do produto comercila $\left(\right.$ Tiger $^{\circledR} 100$ EC) e Espiromesifeno $0,6 \mathrm{~L} \mathrm{ha}^{-1}$ do produto comercial (Oberon ${ }^{\circledR}$ 240 SC) visando mantê-las livres da praga durante todo o estudo. 
As avaliações ocorreram semanalmente, a partir do estádio fenológico V7-V9, aos 9 DAI (dias após a infestação), sendo realizadas até o estádio R5.1R5.3 aos 48 DAI. Nas avaliações foram realizadas a contagem do número de ninfas e ovos em todos os folíolos da planta inteira, sendo separados em terço superior, médio e inferior, em relação à altura da planta (chamado de estudo dos estratos) que foram previamente identificados, com o intuito de acompanhar a flutuação populacional de ovos e ninfas, comparando com a avaliação de todos os folíolos da planta (avaliação da planta inteira), para obtenção do número total de ninfas por cultivar, perfazendo um total de 6 avaliações.

Os índices de produtividade analisados foram: número de vagem por planta, número de grãos por vagem, massa de 100 grãos e massa total de duas plantas. Após a colheita, mediu-se a umidade do grão e a massa foi corrigida para $12 \%$ de umidade.
Os dados foram submetidos à análise de variância e ao teste F de significância $(\alpha<0,05)$ e as médias comparadas pelo teste de Tukey ao nível de $5 \%$ de probabilidade, obtendo-se as diferenças mínimas significativas entre os tratamentos.

\section{RESULTADOS E DISCUSSÃO}

O número de ninfas de $B$. tabaci aumentou na proporção da eclosão, cuja fase de ovo tem duração de 5 a 9 dias (Lopez et al., 2008) (Figs. 1 e 2). Os cultivares BRS 245 RR, BRS 243 RR, BRS 246 RR, BRS 239, CD 202, M 7908 RR e NK 7059 apresentaram número de ninfas inferior a 1.000 na avaliação da planta inteira. Os cultivares CD 214 RR, EMBRAPA 48 e CD 219 RR apresentaram 1.800, 1.063 e 2.128 ninfas, respectivamente, na avaliação do dia 24 de dezembro (Tabela 1).

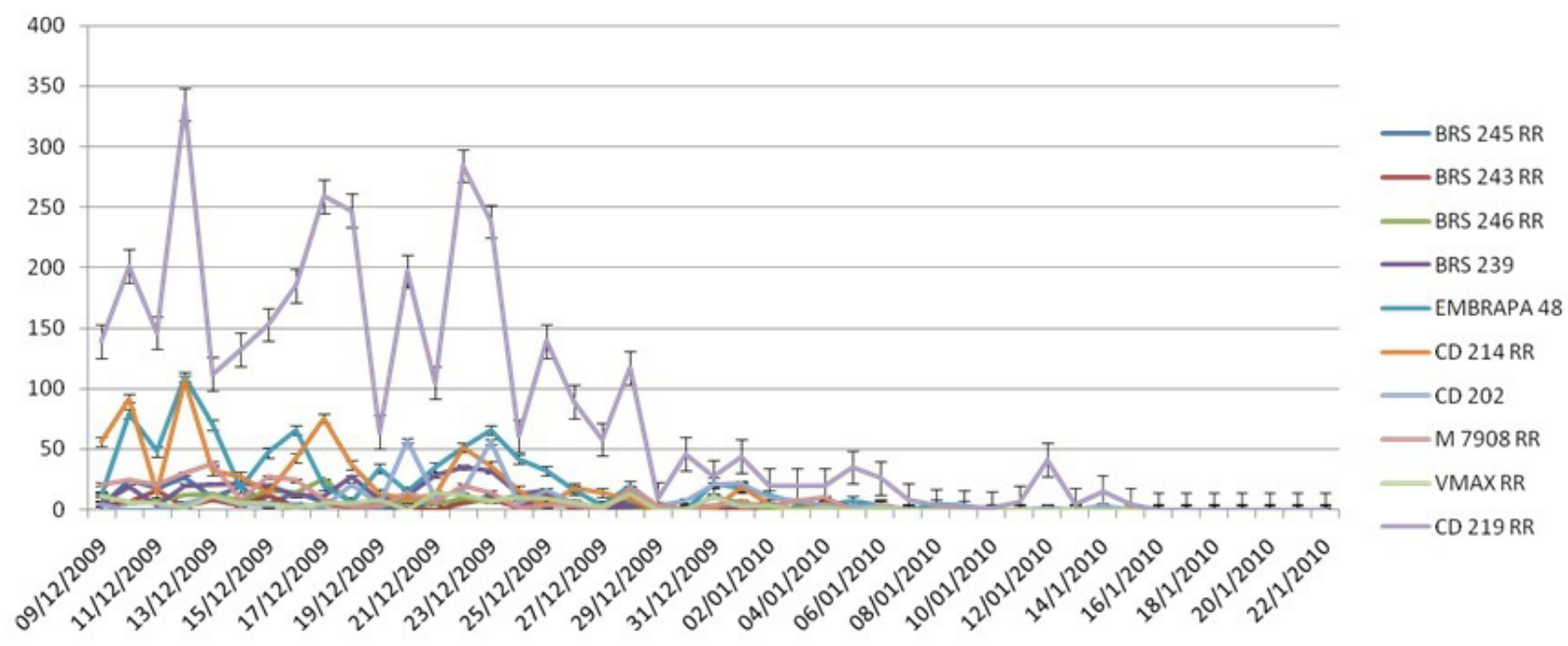

Fig. 1 - Incidência de ovos de mosca-branca nos cultivares de soja e erro padrão em relação à média, em avaliações diárias no período de 9 de dezembro de 2009 até 22 de janeiro de 2010. Dourados, MS, 2010.

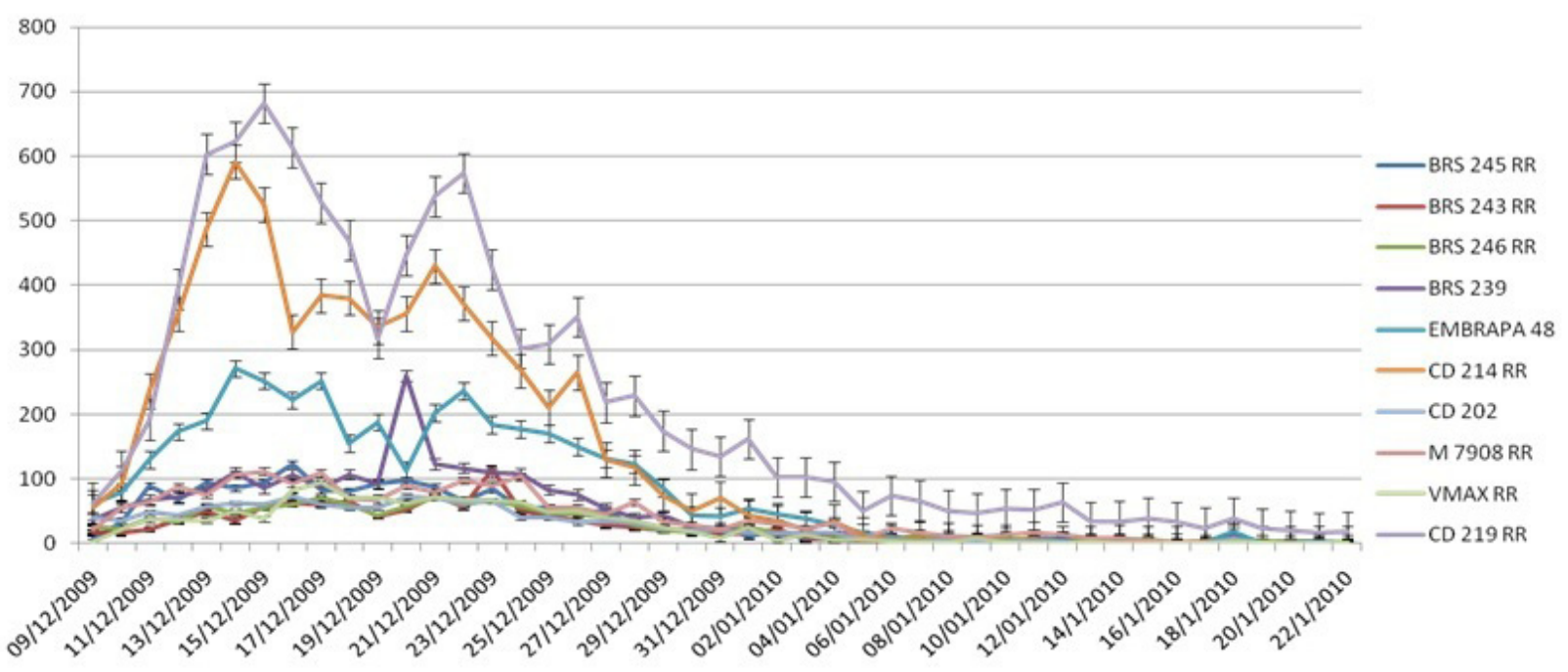

Fig. 2 - Incidência de ninfas de mosca-branca nos cultivares de soja e erro padrão em relação à média, em avaliações diárias no período de 9 de dezembro de 2009 até 22 de janeiro de 2010. Dourados, MS, 2010. 
$\mathrm{Na}$ primeira avaliação realizada aos $9 \mathrm{DAI}$, não houve diferença no número de ninfas entre as cultivares estudadas (Tabela 2). Nas avaliações seguintes ocorreram diferenças $(\mathrm{P}<0,05)$ no número de ninfas ocorrentes nas cultivares de soja. Para variável estrato, diferenças significativas $(\mathrm{P}<0,05)$ foram encontradas nos avaliações de 17 DAI até 41 DAI (Tabela 2).

Para segunda avaliação realizada aos 17 DAÍ, os cultivares Coodetec 219 RR (164,7 ninfas/ planta) e Coodetec 214 RR (118,8 ninfas/planta) apresentaram médias superiores no número de ninfas, ocorrendo diferença significativa $(\mathrm{P}<0,05)$ em relação à cultivar BRS 243 RR (25,4 ninfas/ planta) (Tabela 2).

Em relação à terceira avaliação aos 22 daí, os cultivares Coodetec 219 RR (177,3 ninfas/planta) e Coodetec 214 RR (150,0 ninfas/planta) foram os cultivares que tiveram a maior média de ninfas, diferindo dos cultivares BRS 245 RR, BRS 243 RR, BRS 246 RR e NK 7059 RR, enquanto que os cultivares BRS 239, Embrapa 48, CD 202 e M 7908 RR tiveram médias de ninfas que não diferiram entre si $(\mathrm{P}<0,05)$ a demais variedades (Tabela 2).

Tabela 1 - Incidência de ninfas de mosca-branca na avaliação semanal da planta inteira dos cultivares de soja. Dourados, MS, 2010.

\begin{tabular}{lcccccc}
\hline \multirow{2}{*}{ Cultivares } & \multicolumn{5}{c}{$\mathrm{N}^{\circ}$ de ninfas na planta inteira } \\
\cline { 2 - 7 } & $11 / \mathrm{dez} / 09$ & $19 / \mathrm{dez} / 09$ & $24 / \mathrm{dez} / 09$ & $02 /$ jan/10 & 11/jan/10 & $18 /$ jan/10 \\
\hline BRS 245 RR & 485 & 508 & 454 & 128 & 38 & 36 \\
BRS 243 RR & 280 & 305 & 250 & 120 & 18 & 14 \\
BRS 246 RR & 347 & 496 & 533 & 155 & 28 & 18 \\
BRS 239 & 446 & 596 & 681 & 219 & 25 & 19 \\
EMBRAPA 48 & 864 & 867 & 1.063 & 249 & 355 & 19 \\
CD 214 RR & 725 & 1.426 & 1.800 & 635 & 50 & 20 \\
CD 202 & 578 & 669 & 634 & 188 & 31 & 20 \\
M 7908 RR & 562 & 818 & 828 & 459 & 106 & 25 \\
NK 7059 RR & 209 & 422 & 458 & 187 & 53 & 28 \\
CD 219 RR & 1.052 & 1.976 & 2.128 & 947 & 498 & 264 \\
\hline
\end{tabular}

Tabela 2 - Resumo da análise de variância, média do número de ninfas nos cultivares e nos estratos avaliadas na planta inteira em cada cultivar de soja durante as avaliações. DAI (dias após a infestação). Dourados, MS, 2010.

\begin{tabular}{|c|c|c|c|c|c|c|}
\hline \multirow{2}{*}{ F.V } & \multicolumn{6}{|c|}{ Quadrado médio } \\
\hline & 09 DAI & 17 DAI & $22 \mathrm{DAI}$ & 31 DAÍ & 41 DAÍ & $48 \mathrm{DAI}$ \\
\hline Cultivar & $22,67^{*}$ & $39,22^{*}$ & $74,69^{*}$ & $28,19^{*}$ & $13,83^{*}$ & $7,86^{*}$ \\
\hline Estrato & $11,79^{\text {ns }}$ & $81,16^{*}$ & $106,77^{*}$ & $47,26^{*}$ & $10,89^{*}$ & $2,08^{\mathrm{ns}}$ \\
\hline Cultivar $\mathrm{x}$ estrato & $7,38^{\mathrm{ns}}$ & $11,09^{\text {ns }}$ & $8,16^{\mathrm{ns}}$ & $3,23^{\text {ns }}$ & $2,82^{\mathrm{ns}}$ & $1,34^{\mathrm{ns}}$ \\
\hline \multirow{2}{*}{ Cultivar } & \multicolumn{6}{|c|}{ Média de ninfas/cultivar } \\
\hline & 09 DAI & 17 DAI & 22 DAI & 31 DAÍ & 41 DAÍ & 48 DAI \\
\hline 1 BRS 245 RR & $40,4 \mathrm{a}$ & $42,3 \mathrm{ab}$ & $37,9 \mathrm{a}$ & $10,7 \mathrm{ab}$ & $3,2 \mathrm{a}$ & $3,0 \mathrm{a}$ \\
\hline 2 BRS 243 RR & $23,3 \mathrm{a}$ & $25,4 \mathrm{a}$ & $20,9 a$ & $10,0 \mathrm{a}$ & $1,5 \mathrm{a}$ & $1,2 \mathrm{a}$ \\
\hline 3 BRS 246 RR & $28,9 \mathrm{a}$ & $41,3 \mathrm{ab}$ & $44,4 \mathrm{a}$ & $12,9 \mathrm{ab}$ & $2,3 \mathrm{a}$ & $1,5 \mathrm{a}$ \\
\hline 4 BRS 239 & $37,2 \mathrm{a}$ & $49,7 \mathrm{ab}$ & $56,8 \mathrm{ab}$ & $18,3 \mathrm{ab}$ & $2,1 \mathrm{a}$ & $1,6 \mathrm{a}$ \\
\hline 5 EMBRAPA 48 & $72,0 \mathrm{a}$ & $72,5 \mathrm{ab}$ & $88,6 \mathrm{ab}$ & $20,8 \mathrm{ab}$ & $1,1 \mathrm{a}$ & $1,6 \mathrm{a}$ \\
\hline 6 CD 214 RR & $60,4 \mathrm{a}$ & $118,9 \mathrm{~b}$ & $150,0 \mathrm{~b}$ & $29,6 \mathrm{ab}$ & $4,2 \mathrm{a}$ & $1,7 \mathrm{a}$ \\
\hline 7 CD 202 & $48,2 \mathrm{a}$ & $55,8 \mathrm{ab}$ & $52,9 \mathrm{ab}$ & $15,7 \mathrm{ab}$ & $2,6 \mathrm{a}$ & $1,7 \mathrm{a}$ \\
\hline 8 M 7908 RR & $63,9 a$ & $68,2 \mathrm{ab}$ & $69,0 \mathrm{ab}$ & $38,3 \mathrm{bc}$ & $8,9 \mathrm{a}$ & $2,1 \mathrm{a}$ \\
\hline 9 NK 7059 RR & $25,9 \mathrm{a}$ & $35,2 \mathrm{ab}$ & $38,2 \mathrm{a}$ & $15,6 \mathrm{ab}$ & $4,4 \mathrm{a}$ & $2,3 \mathrm{a}$ \\
\hline 10 CD 219 RR & $87,7 \mathrm{a}$ & $164,7 \mathrm{~b}$ & $177,3 \mathrm{~b}$ & $78,9 \mathrm{c}$ & $41,5 \mathrm{~b}$ & $22,0 \mathrm{~b}$ \\
\hline \multirow{2}{*}{ Estrato } & \multicolumn{6}{|c|}{ Número de ninfas } \\
\hline & 09 DAI & $17 \mathrm{DAI}$ & $22 \mathrm{DAI}$ & 31 DAÍ & 41 DAÍ & $48 \mathrm{DAI}$ \\
\hline Superior & $57,25 \mathrm{a}$ & $81,5 \mathrm{a}$ & $94,72 \mathrm{a}$ & $35,97 \mathrm{a}$ & $12,12 \mathrm{a}$ & $6,07 \mathrm{a}$ \\
\hline Médio & $51,57 \mathrm{a}$ & $83,77 \mathrm{a}$ & $79,52 \mathrm{ab}$ & $25,40 \mathrm{~b}$ & $6,85 \mathrm{ab}$ & $2,80 \mathrm{a}$ \\
\hline Inferior & $37,52 \mathrm{a}$ & $36,8 \mathrm{~b}$ & $46,47 \mathrm{~b}$ & $13,80 \mathrm{~b}$ & $2,52 \mathrm{~b}$ & $2,70 \mathrm{a}$ \\
\hline C.V. (\%) & 51,58 & 54,43 & 50,90 & 46,94 & 80,36 & 68.56 \\
\hline
\end{tabular}

*significativo pelo teste $\mathrm{F}$ a $5 \%$, ns - não significativo. ${ }^{*}$ médias seguidas pela mesma letra, na coluna, não diferem entre si pelo teste Tukey a $5 \%$ de probabilidade. 
Tabela 3 - Média do número de ninfas avaliadas diariamente em 3 folíolos de cada cultivar de soja durante as avaliações. DAI (dias após a infestação). Dourados, MS, 2010.

\begin{tabular}{|c|c|c|c|c|c|c|}
\hline \multirow{2}{*}{ FV } & \multicolumn{6}{|c|}{ Quadrado médio } \\
\hline & 09 DAÍ & 17 DAÍ & $22 \mathrm{DAI}$ & $31 \mathrm{DAI}$ & 41 DAÍ & $48 \mathrm{DAI}$ \\
\hline Cultivares & $1275,84^{\mathrm{ns}}$ & $3107,17^{\text {ns }}$ & $2248,48^{*}$ & $198,01^{*}$ & $64,37^{*}$ & $33,30^{\text {ns }}$ \\
\hline Bloco & 758,75 & 357,73 & 16,00 & 30,03 & 22,96 & 87,42 \\
\hline Resíduo & 1294,57 & 1532,21 & 963,48 & 64,77 & 25,68 & 15,66 \\
\hline \multirow{2}{*}{ Tratamentos } & \multicolumn{6}{|c|}{ Média de ninfas/folíolo } \\
\hline & 09 DAÍ & 17 DAÍ & 22 DAI & $31 \mathrm{DAI}$ & 41 DAÍ & $48 \mathrm{DAI}$ \\
\hline 1 BRS 245 RR & $22,3 \mathrm{a}$ & $23,0 \mathrm{a}$ & $14,0 \mathrm{a}$ & $4,8 \mathrm{a}$ & $2,3 a b$ & $2,8 \mathrm{ab}$ \\
\hline 2 BRS 243 RR & $5,5 \mathrm{a}$ & $10,5 \mathrm{a}$ & $12,8 \mathrm{a}$ & $5,3 \mathrm{a}$ & $0,0 \mathrm{a}$ & $0,8 \mathrm{ab}$ \\
\hline 3 BRS 246 RR & $9,8 \mathrm{a}$ & $10,8 \mathrm{a}$ & $14,3 \mathrm{a}$ & $4,3 \mathrm{a}$ & $1,5 \mathrm{ab}$ & $2,5 \mathrm{ab}$ \\
\hline 4 BRS 239 & $17,0 \mathrm{a}$ & $23,0 \mathrm{a}$ & $27,0 \mathrm{a}$ & $3,3 \mathrm{a}$ & $0,5 \mathrm{a}$ & $3,8 \mathrm{ab}$ \\
\hline 5 EMBRAPA 48 & $32,5 \mathrm{a}$ & $47,0 \mathrm{a}$ & 44,3 a & $11,5 \mathrm{ab}$ & $0,3 \mathrm{a}$ & $4,8 \mathrm{ab}$ \\
\hline 6 CD 214 RR & $59,0 \mathrm{a}$ & $73,8 \mathrm{a}$ & $67,0 \mathrm{a}$ & $9,0 \mathrm{ab}$ & $1,8 \mathrm{ab}$ & $0,5 \mathrm{ab}$ \\
\hline 7 CD 202 & $12,5 \mathrm{a}$ & $14,0 \mathrm{a}$ & $10,3 \mathrm{a}$ & $3,8 \mathrm{a}$ & $0,8 \mathrm{a}$ & $2,3 \mathrm{ab}$ \\
\hline 8 M 7908 RR & $16,0 \mathrm{a}$ & $16,8 \mathrm{a}$ & $26,0 \mathrm{a}$ & $7,8 \mathrm{ab}$ & $4,3 \mathrm{ab}$ & $0,0 \mathrm{a}$ \\
\hline 9 NK 7059 RR & $9,3 \mathrm{a}$ & $17,8 \mathrm{a}$ & $16,0 \mathrm{a}$ & $1,3 \mathrm{a}$ & $0,0 \mathrm{a}$ & $0,8 \mathrm{ab}$ \\
\hline $10 \mathrm{CD} 219 \mathrm{RR}$ & $47,8 \mathrm{a}$ & $79,5 \mathrm{a}$ & $75,5 \mathrm{a}$ & $25,8 \mathrm{~b}$ & $13,3 \mathrm{~b}$ & $9,8 \mathrm{~b}$ \\
\hline CV (\%) & 60,19 & 52,43 & 49,19 & 44,82 & 58,55 & 58,90 \\
\hline
\end{tabular}

*significativo pelo teste $\mathrm{F}$ a $5 \%$, ns - não significativo.

*médias seguidas pela mesma letra, na coluna, não diferem entre si pelo teste Tukey a 5\% de probabilidade.

Para a quarta avaliação aos 31 DAI, o cultivar CD 219 RR continuou com a maior média de ninfas $(78,9$ ninfas/planta), diferindo dos outros cultivares, com exceção do cultivar M 7908 RR que não diferiu da CD 219 RR $(\mathrm{P}<0,05)$ a CD 219 (Tabela 2).

Já na quinta e sexta avaliação, ocorridas, respectivamente, aos 41 e 48 daí, o cultivar Coodetec 219 RR teve a média de 41,5 e 22,0 ninfas por planta (Tabela 2$)$, diferindo $(\mathrm{P}<0,05)$ dos demais cultivares.

Na primeira avaliação realizada uma semana após a infestação do experimento aos 9 DAI não houve diferença significativa $(\mathrm{P}<0,05)$ no número de ninfas entre os estratos superior, médio e inferior (Tabela 2). Isso pode ser explicado pelo fato de ter ocorrido uma baixa população da praga e pelo fato de sua população estar iniciando a multiplicação nos cultivares.

A partir de 17 DAI até a avaliação de 41 DAI, com exceção da avaliação do dia de 31 DAI, as ninfas de $B$. tabaci distribuíram-se verticalmente nas plantas de maneira semelhante, sendo que sua distribuição nos estratos variou significativamente entre o terço superior e o terço médio. Já na a avaliação de 48 DAI, as populações de ninfas igualaram-se entre os estratos avaliados (Tabela 2).

Comportamento semelhante foi observado por LIMA; CAMPOS (2008) trabalhando com a cultura do pimentão. Esta maior preferência pelo estrato superior e médio pode estar relacionada à ocorrência maior conteúdo de açúcares e de nitrogênio (LENTEREN; Noldus, 1990). Para a prática de campo, Degrande;
VIVAN (2010) sugeriram que as amostragens para adultos e ninfas de $B$. tabaci, na cultura da soja, sejam realizadas no terço médio da planta com o intuito de verificar a infestação.

A título de comparação, foi realizada a avaliação da preferência das ninfas nos cultivares estudados com basenas avaliações de 3 folíolos da planta. Nestas avaliações, em que os folíolos foram previamente demarcados, para a avaliação de 9 e 19 DAI não houve diferença significativa do número de ninfas. Já a partir de 22 DAI até a avaliação de 41 DAI houve diferença do número de ninfas. Na última avaliação, aos $48 \mathrm{DAI}$, a população se igualou na avaliação dos folíolos (Tabela 3).

Na avaliação de31DAICD219RR teveuma média de 25,8 ninfas em relação a BRS 245 RR $(4,8)$, BRS 243 RR (5,3), BRS246 RR (4,3), BRS239 (3,3), CD $202(3,8) \mathrm{e}$ NK 7059 RR $(1,3)$. Para a avaliação aos 41 DAI CD 219 RR diferiu de BRS 243 RR, BRS 239, Embrapa 48, CD 202 e NK 7059 RR. Já para a avaliação em 48 DAI CD 219 RR diferiu apenas de M 7908 RR. Ao longo das avaliações, CD 219 RR foi a que apresentou a maior média do número de ninfas na avaliação dos folíolos previamente demarcados (Tabela 3).

Considerando os dados da comparação entre os dados do folíolo e da planta inteira é notável algumas diferenças nos dados coletados. Lima et al. (2002) sugeriram que um folíolo é uma unidade amostral representativa da oviposição do inseto em soja visando facilitar os trabalhos de levantamento e futuras seleções, quando um grande número de genótipos estiver sendo avaliado. Para tal, houve uma 
pequena variação nos resultados desse estudo, sendo que alguns cultivares como a M 7908 RR e BRS 243 RR apresentaram resultados distintos na comparação do tipo de avaliação. Já o cultivar CD 219 RR destacou-se apresentando o maior número de ninfas para ambos os tipos de amostragens. Com isso, a avaliação de todos os folíolos da planta minimiza os riscos das variações na amostragem, porém, quando se trabalha com muitos genótipos, a avaliação torna-se onerosa.

Então, a partir dos dados obtidos indica-se para os estudos em que se trabalha com diversos genótipos de soja, visando a uma amostragem eficiente, sob condições de casa de vegetação, que sejam avaliados todos os folíolos do estrato superior e médio, pois foram nesses locais onde se concentrou a maior quantidade de ninfas de B. tabaci.

Em todas as variáveis-resposta de índices de produtividade estudadas não houve influência do ataque da mosca-branca na comparação das parcelas infestadas com as parcelas testemunha. Para o número de vagens não houve diferença significativa $(\mathrm{P}<$ $0,05)$ entre a cultivar infestada comparada com sua testemunha seminfestação, no entanto, na estimativa de perda em relação à testemunha aponta danos no cultivar CD 202 (16,5\%), M 7908 RR (15,8\%). Nota-se que BRS 243 RR e NK 7059 RR não tiveram danos no número de vagem em relação à parcela testemunha. Nos demais cultivares as reduções variaram entre $0,7 \%$ a $13,1 \%$ (Tabela 4 ).

Não houve diferença significativa $(\mathrm{P}<0,05)$ para o número de grãos; entretanto, na estimativa de perda em relação à testemunha, BRS 246 RR foi prejudicado em $38,5 \%$ em relação a sua testemunha, seguido de CD 202 (15,6\%), CD 214 RR (14,9\%), M
7908 RR (12,8\%), EMBRAPA 48 (8,5\%), BRS 245 RR (6,4\%), BRS $239(4,2 \%)$, BRS $243(2,6 \%)$ e CD 219 RR $(1,5 \%)$. NK 7059 RR não foi afetado pelo ataque da mosca-branca (Tabela 4).

Para a massa total também não houve diferença significativa $(P<0,05)$, todavia, na estimativa de perda M 7908 RR foi prejudicada em 20,3\% na produção, seguido de CD 202 (18,1\%), BRS 246 RR (17,9\%), CD 214 RR (12,9\%), EMBRAPA 48 (10,5\%), BRS $239(5,8 \%)$, CD 219 RR (4\%) e BRS 245 RR $(2,9 \%)$. Segundo essa estimativa, os cultivares NK 7059 RR e BRS 243 RR não foram afetadas pelo ataque da praga para massa total (Tabela 4).

Não houve influência significativa $(P<0,05)$ para massa de 100 grãos nos cultivares, contudo, na estimativa de perda destacam-se M 7908 RR (8\%), seguido de NK 7059 RR (4,6\%), CD 202 (3,6\%), BRS 239 (1,7\%), CD 219 RR (1,6\%), EMBRAPA 48 (0,3\%) de perdas em relação a sua testemunha. Os cultivares BRS 245 RR BRS 243 RR, BRS 246 RR e CD 214 RR não tiveram perdas em relação à testemunha (Tabela 4).

Dentre os cultivares de soja avaliadas, existem cultivares maisfavoráveis para a mosca-branca comoa CD 219RR coma infestação máxima de2.128 ninfas na avaliação da planta inteira (Tabela1). Odano ocorrido na parcela foi de apenas $4 \%$ de perda em relação a sua testemunha (Tabela 4). Por outro lado, o cultivar BRS 246RR apresentou uma infestação significativamente inferior a CD 219 RR, com 17,9\% de perda em relação a sua parcela testemunha. Observa-se nos cálculos de estimativas de perdas que o cultivar NK 7059 RR não teve prejuízos em relação à testemunha para as variáveis número de vagens, número de grãos emassa total de duas plantas.

Tabela 4 - Análise dos fatores produtivos dos cultivares submetidos à infestação artificial da mosca-branca (2 plantas). Sem infestação (SI), com infestação (CI) e porcentagem de perda em relação à testemunha. Dourados, MS, 2010.

\begin{tabular}{|c|c|c|c|c|c|c|c|c|c|c|c|c|}
\hline \multirow{3}{*}{ Tratamentos } & \multicolumn{12}{|c|}{ Fatores produtivos/parcela } \\
\hline & \multicolumn{3}{|c|}{$\mathrm{N}^{\circ}$ vagem } & \multicolumn{3}{|c|}{$\mathrm{N}^{\circ}$ grãos } & \multicolumn{3}{|c|}{ Massa total 2 plantas } & \multicolumn{3}{|c|}{ Massa 100 grãos } \\
\hline & SI & $\mathrm{CI}$ & $\%$ & SI & $\mathrm{CI}$ & $\%$ & SI & $\mathrm{CI}$ & $\%$ & SI & $\mathrm{CI}$ & $\%$ \\
\hline BRS 245 RR & $135,5 \mathrm{~A}$ & 132,3 a & 2,4 & $261,8 \mathrm{~A}$ & $245,1 \mathrm{a}$ & 6,4 & $32,9 \mathrm{~A}$ & $31,9 \mathrm{a}$ & 2,9 & $12,8 \mathrm{a}$ & $13,1 \mathrm{a}$ & $-2,6$ \\
\hline BRS 243 RR & $114,5 \mathrm{~A}$ & 124,8 a & $-9,0$ & $251,2 \mathrm{~A}$ & 244,8 a & 2,6 & $33,6 \mathrm{~A}$ & $34,3 \mathrm{a}$ & $-2,1$ & $13,3 \mathrm{a}$ & $13,4 \mathrm{a}$ & $-0,5$ \\
\hline BRS 246 RR & $139,3 \mathrm{~A}$ & $121,0 \mathrm{a}$ & 13,1 & $369,3 \mathrm{~A}$ & $227,1 \mathrm{a}$ & 38,5 & $36,2 \mathrm{~A}$ & $29,7 \mathrm{a}$ & 17,9 & $12,4 \mathrm{a}$ & $13,1 \mathrm{a}$ & $-5,9$ \\
\hline BRS 239 & $111,3 \mathrm{~A}$ & $109,0 \mathrm{a}$ & 2,0 & $218,6 \mathrm{~A}$ & $209,3 \mathrm{a}$ & 4,2 & $35,7 \mathrm{~A}$ & 33,6 a & 5,8 & $16,3 \mathrm{a}$ & $16,1 \mathrm{a}$ & 1,7 \\
\hline EMBRAPA 48 & $112,3 \mathrm{~A}$ & $111,5 \mathrm{a}$ & 0,7 & $218,8 \mathrm{~A}$ & $200,2 \mathrm{a}$ & 8,5 & $32,4 \mathrm{~A}$ & 29,0 a & 10,5 & $14,7 \mathrm{a}$ & $14,6 \mathrm{a}$ & 0,3 \\
\hline CD 214 RR & $145,8 \mathrm{~A}$ & $133,3 \mathrm{a}$ & 8,6 & $269,5 \mathrm{~A}$ & $229,4 \mathrm{a}$ & 14,9 & $34,6 \mathrm{~A}$ & $30,2 \mathrm{a}$ & 12,9 & $12,9 \mathrm{a}$ & $13,2 \mathrm{a}$ & $-2,5$ \\
\hline CD 202 & $136,0 \mathrm{~A}$ & $113,5 \mathrm{a}$ & 16,5 & $246,3 \mathrm{~A}$ & 207,8 a & 15,6 & $39,4 \mathrm{~A}$ & $32,3 \mathrm{a}$ & 18,1 & $16,2 \mathrm{a}$ & $15,6 \mathrm{a}$ & 3,6 \\
\hline M 7908 RR & $96,3 \mathrm{~A}$ & $81,0 \mathrm{a}$ & 15,8 & $190,5 \mathrm{~A}$ & $166,1 \mathrm{a}$ & 12,8 & $32,2 \mathrm{~A}$ & $25,6 \mathrm{a}$ & 20,3 & $16,8 \mathrm{a}$ & $15,5 \mathrm{a}$ & 8,0 \\
\hline NK 7059 RR & $96,3 \mathrm{~A}$ & $121,0 \mathrm{a}$ & $-25,7$ & $193,8 \mathrm{~A}$ & $262,5 \mathrm{a}$ & $-35,4$ & $29,8 \mathrm{~A}$ & $38,2 \mathrm{a}$ & $-28,3$ & $15,4 \mathrm{a}$ & $14,7 \mathrm{a}$ & 4,6 \\
\hline CD 219 RR & $144,5 \mathrm{~A}$ & $133,8 \mathrm{a}$ & 7,4 & $292,2 \mathrm{~A}$ & $287,7 \mathrm{a}$ & 1,5 & $37,4 \mathrm{~A}$ & 35,9 a & 4,0 & $12,8 \mathrm{a}$ & $12,6 \mathrm{a}$ & 1,6 \\
\hline $\mathrm{CV}(\%)$ & 21,33 & 15,60 & & 26,64 & 17,60 & & 20,00 & 16,50 & & 8,79 & 8,00 & \\
\hline F (Trats) & $5,96^{\mathrm{ns}}$ & $1,42^{*}$ & & $3,15^{*}$ & $1,15^{*}$ & & $7,00^{\mathrm{ns}}$ & $9,45^{\mathrm{ns}}$ & & $0,00 *$ & $0,00^{*}$ & \\
\hline
\end{tabular}

*significativo pelo teste $\mathrm{F}$ a $5 \%$, ns - não significativo.

*médias seguidas pela mesma letra para cada variável analisada, na linha, não diferem entre si pelo teste Tukey a 5\% de probabilidade. 


\section{CONCLUSÕES}

O cultivar de soja CD 219 RR foi a mais infestada por ninfas de B. tabaci;

A maior incidência de ninfas de mosca-branca ocorreu nos terços superior e médio das plantas definidos como locais amostrais em ensaios com plantas de soja nas condições de casa de vegetação;

$\mathrm{O}$ ataque de $B$. tabaci não afetou os índices de produtividade dos cultivares estudados.

\section{REFERÊNCIAS}

ASSOCIAÇÃO BRASILEIRA DAS INDÚSTRIAS DE ÓLEOS VEGETAIS Estatística mensal ano safra 2010/11. São Paulo: ABIOVE, 2010. Seção complexo soja - estatística mensal ano safra 2010/11. Disponível em: <http:/ / www.abiove.com.br/estatistica_br.html> Acesso em: 14 dez. 2010.

ANDERSON, P.; MORALES F. Whitefly and whitefly borne viruses in the tropics: building a knowledge-base for global action. Centro Internacional de Agricultura Tropical (CIAT), v.1, n.341, p.345, 2005.

AZEVEDO, F.R.; BLEICHER, E. Distribuição vertical e setorial das ninfas de mosca-branca nas folhas de meloeiro. Horticultura Brasileira, v.21, p.464-467, 2003.

BUENO, A.F.; BUENO, R.C.O.; FERNANDES, O.A. Resposta fisiológica das plantas de soja e tomate à injúria causada pela mosca-branca Bemisia tabaci raça $\mathrm{B}$ (Hemiptera: Aleyrodidae). Ecossistema, v.30, n.2, p.45-49, 2006.

CHU, C.C. FREEMAN, T.P.; BUCKNER, J.S.; HENNEBERRY, T.J.; NELSON, D.R.; NATWICK, E.T. Susceptibility of upland cotton cultivars to Bemisia tabaci biotype B (Homoptera: Aleyrodidae) in relation to leaf age and trichome density. Annals of the Entomological Society of America, v.94, n.5, p.743-749, 2001.

\section{CONSELHO NACIONAL DE ABASTECIMENTO}

(Brasil). Acompanhamento de safra brasileira: grãos, segundo levantamento, setembro 2012. Brasília. Disponível em: $<$ http://www.conab.gov.br/OlalaCMS/uploads/arquivos/12_12_06_09_10_01_boletim_portugues_dezembro_2012.pdf > Acesso em: 17 abr. 2013.

DEGRANDE, P.E.; VIVAN, L.M. Devastadoras. Cultivar Grandes Culturas, v.1, n.71, p.20-22, 2005.

DEGRANDE, P.E.; VIVAN, L.M. Pragas da soja. Boletim de pesquisa de soja da Fundação MT, v.1, n.14, p.152-215, 2010.

DIEHL, J.; ELLSWORTH, P.; NARANJO, S. Whiteflies in Arizona: binomial sampling of nymphs. Tucson: University of Arizona, 1997a. 2p. (Cooperative Extension

Series, 11).

DIEHL, J.W.; NARANJO, S.E.; ELLSWORTH, P.C. Whitefly growth regulators: a field sampling protocol for nymphs. In: Cotton: A College of Agriculture Report. Tucson: University of Arizona, 1997b. p 265-271.

(Cooperative Extension, Series P-108).

FEHR, W.R.; CAVINESS, C.E. Stages on soybean development. Ames: Iowa State University, 1977. 12p. (Special Report, 80.

FLINT, H.M.; PARKS, N.J. Infestation of germoplasm lines and cultivars of cotton in Arizona by whitefly nymphs (Homoptera: Aleyrodidae). Journal of Entomological Science, v.25, n.2, p.223-229, 1990.

GOMES, F. P. Curso de estatística experimental. São Paulo: Nobel, 1982. v.12, n.1.

HAJI, F.N.P.; CARNEIRO, J. da S.; BLEICHER, E.; MOREIRA, A.N.; FERREIRA, R.C.F. Manejo da mosca-branca Bemisia tabaci biótipo B na cultura do tomate. In: HAJI, F.N.P.; BLEICHER, E. (Ed.). Avanços no manejo da mosca-branca Bemisia tabaci biótipo B (Hemiptera, Aleyrodidae). Petrolina, PE: Embrapa Semi-Árido, 2004. cap. 7, p.87-110.

LENTEREN, J.C. VAN; NOLDUS, L.P.J.J. Whiteflyplant relationships: behavioural and ecological aspects. Whiteflies, v.1, n.1, p.47-89, 1990.

LIMA, A.C.S.; CAMPOS, A.R. Fatores que afetam a oviposição de Bemisia tabaci (Genn.) biótipo B (Hemiptera: Aleyrodidae) em pimentão. Neotropical Entomology, v.37, n.2, p.180-184, 2008.

LIMA, A.C.S.; LARA, F.M.; BARBOSA, J.C. Preferência para oviposição de Bemisia tabaci (Genn.) Biótipo B (Hemíptera: Aleyrodidae) em genótipos de soja, sob condições de campo. Neotropical Entomology, v.31, n.1, p.297-303, 2002.

LOPEZ V.; VOS, J.; POLAR, P.; KRAUSS, U. Discovery learning about sustainable management of whitefly pests and whitefly-borne viruses.

International Centre for Tropical Agriculture (CIAT), v.1, n.1, p.12-37, 2008.

LOURENÇÃO, A.L.; NAGAI, H. Surtos populacionais de Bemisia tabaci no estado de São Paulo. Bragantia, v.53, p.53-59. 1994.

LOURENÇÃO, A.L.; SOUSA-DIAS, J.A.C.; FUGI, C.G.Q. Mosca-branca: superpraga da agricultura mundial ameaça a bataticultura brasileira. Batata Show, v.6, n.1, p.7-8, 2003.

VALLE, G.E.; LOURENÇÃO, A.L. Resistência de genótipos de soja a Bemisia tabaci (Genn.) biótipo B 
(Hemiptera: Aleyrodidae). Neotropical Entomology, v.31, n.1, p.285-295, 2002.

VALLE, G.E.; LOURENÇÃO, A.L. Adult attractiveness and oviposition preference of Bemisa tabaci biotype B in soybean genotypes with different trichoma density. Journal of Pest Science, v.85, n.1, p.431-442, 2012.

Recebido em 23/2/12

Aceito em 17/4/13 